\title{
Brønsted Acid-Catalyzed Stereospecific Dearomative Spirocyclization of Benzothiophenyl Analogues of Tertiary cis- $\beta$-Benzylstyrenes
}

\author{
Anargul Tohti, Victoria Lerda, and Benjamin J. Stokes ${ }^{\dagger *}$ \\ Department of Chemistry \& Chemical Biology, University of California, 5200 N. Lake Road, Merced, CA 95343, USA
}

\begin{abstract}
Substituted benzothiophenyl analogues of tertiary cis- $\beta$-benzylstyrenes undergo triflic acid-catalyzed dearomative spirocyclization to afford vicinal quaternary center-containing compounds at room temperature. Hydroarylation of the styrene is a competing process that could be selected for in substrates possessing electron-rich styrenyl alkenes, or an indole in place of the benzothiophene.
\end{abstract}

The construction of quaternary carbons is an area of intense research interest in organic synthesis due to both the prevalence of quaternary centers in biologically active natural products and pharmaceutical agents as well as the challenge of forging congested C-C bonds. ${ }^{1-3}$ Synthetically, quaternary carbons can enhance rates of cyclization reactions and associated selectivity outcomes thereof. ${ }^{4}$ Medicinally, quaternary carbons are relatively metabolically stable. ${ }^{5}$ Among quaternary carbon-containing molecules, spirocycles have garnered particular medicinal attention in recent years ${ }^{6}$ and may be considered a privileged molecular motif in drug discovery as diversifying lynchpins and thanks to their improved pharmaceutical properties compared to aspirocyclic or planar aromatic molecules. ${ }^{7}$ Dearomatization is likely the most common strategy by which spirocycles are prepared, $, 8,9$ including a few examples of Brønsted acid-catalyzed approaches. ${ }^{10}$ Syntheses of spirocycles are constrained by functional group incompatibility and the consequent limited functionality available for subsequent synthetic manipulation. ${ }^{11}$ Additionally, spirocyclizations that furnish vicinal all-carbon quaternary centers have not been reported to the best of our knowledge.

Stemming from our interest in catalytic intramolecular arylations of congested alkenes, ${ }^{12}$ particularly the cis-specific behavior of indole $\mathbf{A}$ (Scheme 1A), ${ }^{12 b}$ we observed that a benzothiophenyl analogue (1a, Scheme 1B) underwent dearomative spirocyclization to form 2a with high chemoselectivity over styrene hydroarylation products including 3a, although styrene hydroarylation is favored for electron-rich styrenes. Herein, a variety of spiroheterocycles bearing vicinal quaternary centers and an intact alkene are prepared from the cis-configured isomers of benzo $[b]$ thiophene derivatives of $\mathbf{1 a}$ through dearomative spirocyclization at room temperature with catalytic triflic acid.
Scheme 1. Brønsted Acid-Catalyzed Stereospecific Intramolecular Hydroarylation Reactions of Heteroaromatic Analogues of Tertiary $\beta$-Benzyl Styrenes.

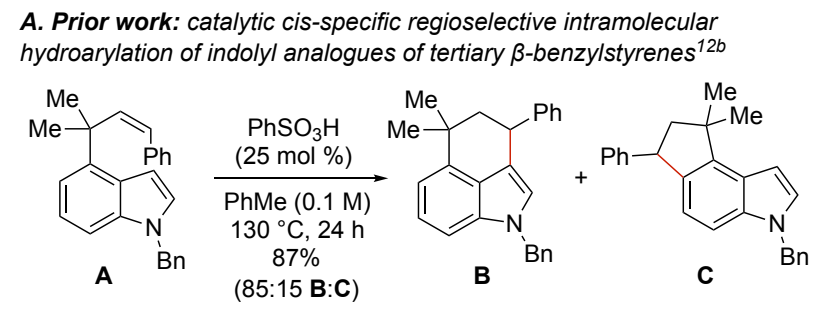

B. This work: catalytic cis-specific regioselective dearomative spirocyclization of benzothiophenyl analogues of tertiary $\beta$-benzylstyrenes

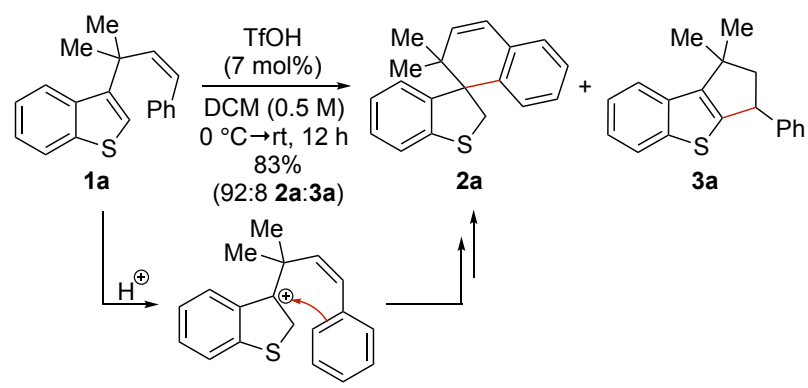

Reaction optimization (see the Supporting Information for details) revealed that spirocyclization could be accomplished in good yield using trifluoromethanesulfonic acid (TfOH) as a catalyst after 12 hours at ambient temperature in dichloromethane. We examined the substrate scope by decorating with various functional groups (Table 1). Catalytic triflic acid afforded dearomative spirocyclized products in good or excellent yield in many cases. For example, meta-substituted styrenes bearing $\mathrm{F}, \mathrm{Cl}, \mathrm{Br}$ or $\mathrm{Me}(\mathbf{1 b}-\mathbf{1 e})$ afforded dearomative spirocycles $\mathbf{2 b}-\mathbf{2 e}$ in high yield with the new bond formed para to the functional group. Of note, $m$-fluoro substrate $\mathbf{2 b}$ afforded the best yield and chemoselectivity that we observed in this study. ${ }^{13}$ Variation of substituents para to the alkene revealed that the chemoselectivity could be inverted by electronic enrichment. Specifically, $p$-methyl substrate $\mathbf{1 f}$ afforded a 65:35 mixture of $\mathbf{2 f}$ and 3f, while $p$-phenyl analogue $\mathbf{1 g}$ afforded a 40:60 ratio of $\mathbf{2 g}$ and $\mathbf{3 g}$, and most impressively $p$-methoxy substrate $\mathbf{1 h}$ selectively afforded the styrene hydroarylation product $\mathbf{3 h}$ in $77 \%$ isolated yield. Naphthyl analogue 1i afforded $46 \%$ yield of $\mathbf{2 i}$ at the less sterically hindered position. We also 


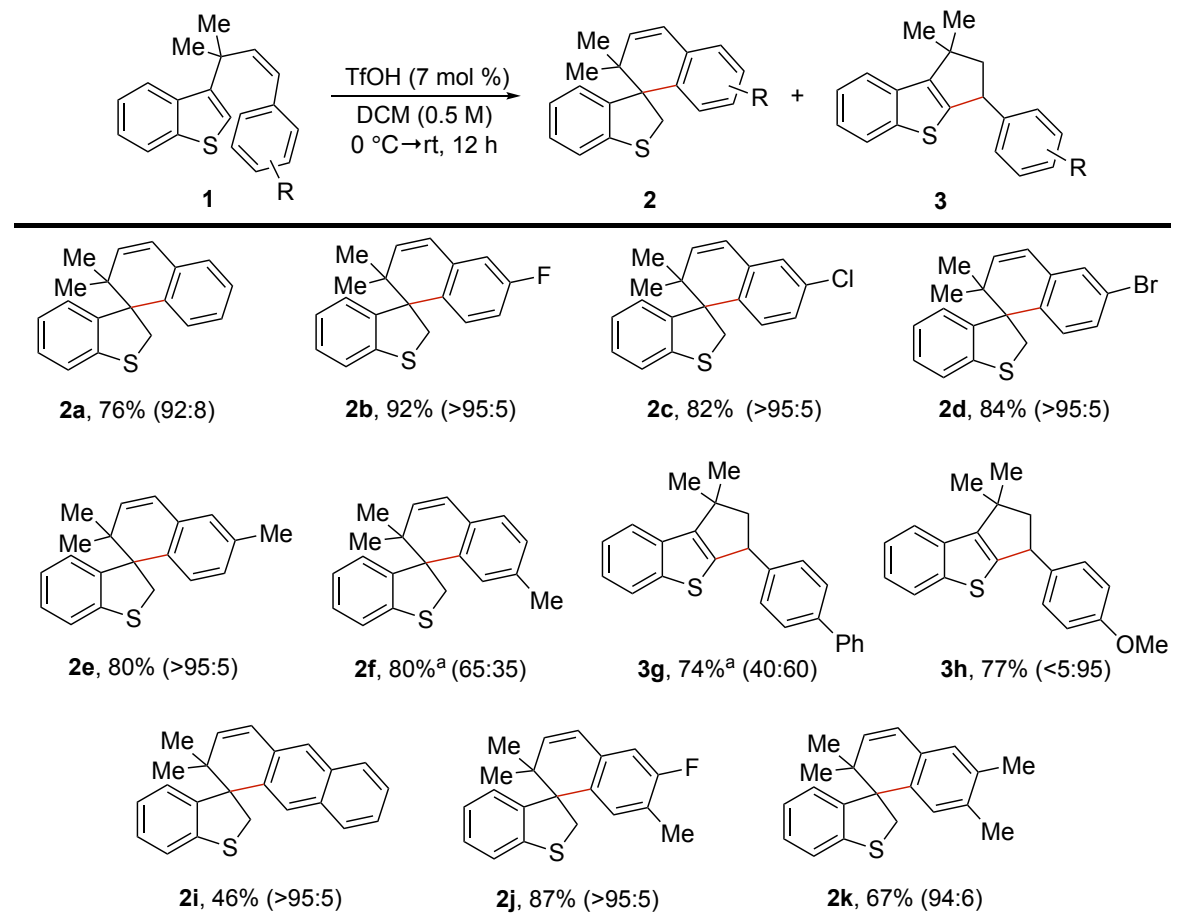

Table 1. Reaction Scope. Reactions were conducted on $0.3 \mathrm{mmol}$ scale in a closed vial. The substrates were fully consumed in all cases. Unless otherwise noted, yields refer to the isolated amount of the indicated major product. Isomeric ratios (2:3, indicated in parentheses) were determined by ${ }^{1} \mathrm{H}$ NMR analysis of the crude reaction mixture. a Combined isolated yield of inseparable regioisomers.

evaluated two disubstituted styrenes, and fluoromethyl substrate $\mathbf{1 j}$ afforded $\mathbf{2} \mathbf{j}$, which possesses the new $\mathrm{C}-\mathrm{C}$ bond para to the F group. The 3,4-dimethyl analogue $\mathbf{1 k}$ afforded $67 \%$ yield of $\mathbf{2 k}$.

The analogous 3-substituted indole and benzofuran analogues of benzothiophene do not undergo spirocyclization appreciably. Rather, indole substrate $\mathbf{4}$ afforded the hydroarylation product 5 in 39\% NMR yield (eq 1), while benzofuran 6 decomposed unproductively (eq 2).

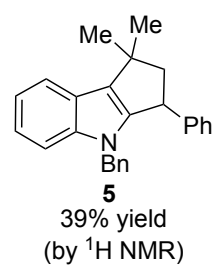

oligomeric decomposition<smiles>CC(C)(/C=C\c1ccccc1)c1c[nH]c2ccccc12</smiles><smiles>CC(C)(/C=C\C(=O)OCC(=O)OCCOc1ccccc1)c1coc2ccccc12</smiles>

In summary, we have developed a Brønsted acid-catalyzed intramolecular dearomative hydroarylation of benzothiophenyl analogues of tertiary cis- $\beta$-benzylstyrenes to access spirocyclic benzocycloalkanes. This is the first example of a spirocyclization furnishing vicinal quaternary centers that we are aware of. Hydroarylation of the styrene is a competing process that predominates for electron-rich styrenyl alkenes.

\section{AUTHOR INFORMATION}

\section{Corresponding Author}

tCurrent address: Department of Chemistry \& Biochemistry, Santa Clara University, 500 El Camino Real, Santa Clara, CA 95053

*bstokes@scu.edu

Notes

The authors declare no competing financial interest.

\section{ACKNOWLEDGMENT}

This research was sponsored by the University of California, Merced and an award from the Hellman Faculty Fellows Fund.

\section{REFERENCES}

(1) For reviews, see: (a) Trost, B. M.; Jiang, C. Synthesis 2006, 3, 369. (b) Christoffers, J.; Mann, A. Angew. Chem. Int. Ed. 2001, 40, 4591.

(c) Büschleb, M.; Dorich, S.; Hanessian, S.; Tao, D.; Schenthal, K. B.; Overman, L. E. Angew. Chem. Int. Ed. 2016, 55, 4156. (d) Ling, T.; Rivas, F. Tetrahedron, 2016, 72, 6729. (e) Li, C.; Ragab, S. S.; Liu, G.; Tang, W. Nat. Prod. Rep. 2020, 37, 276. (f) Corey, E. J.; Guzman-Perez, A. Angew. Chem. Int. Ed. 1998, 37, 388. (g) Das, J. P.; Marek, I. Chem. Commun. 2011, 47, 4593. (h) Hawner, C.; Alexakis, A. Chem. Commun. 2010, 46, 7295. (i) Hong, A. Y.; Stoltz, B. M. Eur. J. Org. Chem. 2013, 14, 2745. (j) Douglas, C. J.; Overmann, L. E. Proc. Natl Acad. Sci. USA 2004, 101, 5363. (k) Feng, J.; Holmes, M.; Krische, M. J. Chem. Rev. 2017, 117, 12564. (l) Cozzi, P. G.; Hilgraf, R.; Zimmermann, N. Eur. J. Org. Chem. 2007, 5969. (m) Long, R.; Huang, J.; Gong, J.; Yang, Z. Nat. Prod. Rep. 2015, 32, 1584. (n) Zhou, F.; Zhu, L.; Pan, B. -W.; Shi, Y.; Liu, Y. -L.; Zhou, L. Chem. Sci. 2020, 11, 9341. (2) For selected approaches, see: (a) Mei, T. -S.; Patel, H. H.; Sigman, M. S. Nature 2014, 508, 340. (b) Trost, B. M.; Malhotra, W. H. Chan, W. H. J. A. Chem. Soc. 2011, 133, 7328. (c) Pierrot, D.; Marek, I. Angew. Chem. Int. Ed. 2020, 59, 36. (d) Bruffaerts, J.; Pierrot, D.; Marek, 
I. Nat. Chem. 2018, 10, 1164. (e) Hu, P.; Chi, H. M.; DeBacker, K. C.; Gong, X.; Keim, J. H.; Hsu, I. T.; Snyder, S. A. Nature 2019, 569, 703. (f) Ohmatsu, K. Imagawa, N.; Ooi, T. Nat. Chem. 2014, 6, 47. (g) Krautwald, S.; Sarlah, D.; Schafroth, M. A.; Carreira, E. M. Science 2013, 340, 1065. (h) Behenna, D. C.; Stoltz, B. M. J. Am. Chem. Soc., 2004, 126, 15044. (i) Zhang, P.; Le, H.; Kyne, R. E.; Morken, J. P. J. Am. Chem. Soc. 2011, 133, 9716. (j) Jung, B.; Hoveyda, A. H. J. Am. Chem. Soc. 2012, 134, 1490.

(3) (a) Quasdorf, K. W.; Overman, L. E. Nature 2014, 516, 181. (b) Liu, Y.; Han, S.; Liu, W.-Bo.; Stoltz, B. M. Acc. Chem. Res. 2015, 48, 740.

(4) Jung, M. E.; Piizzi, G. Chem. Rev. 2005, 105, 1735.

(5) Talele, T. T. J. Med. Chem. 2018, 61, 2166.

(6) (a) Marson, C. M. Chem. Soc. Rev. 2011, 40, 5514. (b) Zheng, Y.; Tice, C. M.; Singh, S. B. Bioorg. Med. Chem. Lett. 2014, 24, 3673. (c) Welsch, M. E.; Snyder, S. A.; Stockwell, B. R. Curr. Opin. Chem. Biol. 2010, 14, 347. (d) Galloway, W. R. J. D.; Isidro-Llobet, A.; Spring, D. R. Nat. Commun. 2010, 1, 80. (e) Sharma, I.; Tan, D. S. Nat. Chem. 2013, 5, 157. (f) Hung, A. W.; Ramek, A.; Wang, Y.; Kaya, T.; Wilson, J. A.; Clemons, P. A.; Young, D. W. Proc. Natl. Acad. Sci. USA. 2011, 108, 6799. (g) Carreira, E. M.; Fessard, T. C. Chem. Rev. 2014, 114, 8257.

(7) (a) Frank, L.; Jack, B.; Christine, H. J. Med. Chem. 2009, 52, 6752. (b) Galliford, C. V.; Scheidt, K. A. Angew. Chem. Int. Ed. 2007, 46, 8748. (c) Müller, G.; Berkenbosch, T.; Benningshof, J. C. J.; Stumpfe, D.; Bajorath, J. Chem. Eur. J. 2017, 23, 703. (d) King, T. A.; Stewart, H. L.; Mortensen, K. T.; North, A. J. P.; Sore, H. F.; Spring, D. R. Eur. J. Org. Chem. 2019, 5219. (e) Stotani, S.; Lorenz, C.; Winkler, M.; Medda, F.; Picazo, E.; Ortega Martinez, R.; Karawajczyk, A.; SanchezQuesada, J.; Giordanetto, F. ACS Comb. Sci. 2016, 18, 330.

(8) For reviews, see: (a) Rios, R. Chem. Soc. Rev., 2012, 41, 1060. (b) Xu, P. W.; Yu, J. S.; Chen, C.; Cao, Z. Y.; Zhou, F.; Zhou, J. ACS Catal. 2019, 9, 1820. (c) Sannigrahi, M. Tetrahedron 1999, 55, 9007. (d) James, M. J.; O’Brien, P.; Taylor, R. J. K.; Unsworth, W. P. Chem. Eur. J. 2016, 22, 2856. (e) D'yakonov, V. A.; Trapeznikova, O. A.; de Meijere, A.; Dzhemilev, U. M. Chem. Rev. 2014, 114, 5775. (f) Liang, X.W.; Zheng, C.; You, S.-L. Chem. -Eur. J. 2016, 22, 11918. (g) Roche, S. P.; Youte Tendoung, J.-J.; Tréguier, B. Tetrahedron 2015, 71, 3549. (h) Zhuo, C.-X.; Zhang, W.; You, S.-L. Angew. Chem. Int. Ed. 2012, 51, 12662. (i) Zhuo, C. X.; Zheng, C.; You, S.-L. Acc. Chem. Res. 2014, 47, 2558. (j) Ding, Q.; Zhou, X.; Fan, R. Org. Biomol. Chem. 2014, 12, 4807. (k) Wertjes, W. C.; Southgate, E. H.; Sarlah, D. Chem. Soc. Rev. 2018, 47, 7996.
(9) Selected examples: (a) Vo, N. T.; Pace, R. D. M.; O'Har, F.; Gaunt, M. J. J. Am. Chem. Soc. 2008, 130, 404. (b) Rousseaux, S.; GarcíaFortanet, J.; Del Aguila Sanchez, M. A.; Buchwald, S. L. J. Am. Chem. Soc. 2011, 133, 9282. (c) Rudolph, A.; Bos, P. H.; Meetsma, A.; Minnaard, A.; Feringa, B. L. Angew. Chem. Int. Ed. 2011, 50, 5834. (d) James, M. J.; Cuthbertson, J. D.; O'Brien, P.; Taylor, R. J. K.; Unsworth, W. P. Angew. Chem. Int. Ed. 2015, 54, 7640. (e) Huang, H. -M.; Procter, D. J. J. Am. Chem. Soc. 2017, 139, 1661. (f) Wu, Q. -F.; He, H.; Liu, W. -B.; You, S. -L. J. Am. Chem. Soc. 2010, 132, 11418. (g) Cai, Q.; Zheng, C.; Zhang, J. -W.; You, S. -L. Angew. Chem. Int. Ed. 2011, 50, 8665. (h) Singh, R. P.; Das, J.; Yousufuddin, M.; Gout, D.; Lovely, C. J. Org. Lett. 2017, 19, 4110. (i) Schröder, F.; Sharma, U. K.; Mertens, M.; Devred, F.; Debecker, D. P.; Luque, R.; Van der Eycken, E. V. ACS Catal. 2016, 6, 8156. (j) Nandi, R.-K.; Guillot, R.; Kouklovsky, C.; Vincent, G. Org. Lett. 2016, 18, 1716. (k) Adams, K.; Ball, A. K.; Birkett, J.; Brown, L.; Chappell, B.; Gill, D. M.; Lo, P. K. T.; Patmore, N. J.; Rice, C. R.; Ryan, J.; Raubo, P.; Sweeney, J. B. Nat. Chem. 2017, 9, 396. (l) Bansode, A. H.; Shaikh, S. R.; Gonnade, R. G.; Patil, N. T. Chem. Commun. 2017, 53, 9081. (m) Mu, X.; Yu, H.; Peng, H.; Xiong, W.; Wu, T.; Tang, W. Angew. Chem. Int. Ed. 2020, 59, 8143. (n) Vacala, T. L.; Carlson, P. R.; Arreola-Hester, A.; Williams, C. G.; Makhoul, E. W.; Vadola, P. A. J. Org. Chem. 2018, 83, 1493. (o) Liddon, J. T. R.; Clarke, A. K.; Taylor, R. J. K.; Unsworth, W. P. Org. Lett. 2016, 18, 6328. (p) Clarke, A. K.; James, M. J.; O'Brien, P.; Taylor, R. J. K.; Unsworth, W. P. Angew. Chem. Int. Ed. 2016, 55, 13798. (q) Wu, T.; Zhou, Q.; Tang, W. Angew. Chem. Int. Ed. 2021, 60, 9978.

(10) (a) Fedoseev, P.; Van Der Eycken, E. Chem. Commun. 2017, 53, 7732. (b) Kong, L.; Sun, Y.; Zheng, Z.; Tang, R.; Wang, M.; Li, Y. Org. Lett. 2018, 20, 5251. (c) Zhou, Y.; Xia, Z. -L.; Gu, Q.; You, S. -L. Org. Lett. 2017, 19, 762. (d) Pan, Z.; Liu, Y.; Hu, F.; Liu, Q.; Shang, W.; Ji, Xu.; Xia, C. Org. Lett. 2020, 22, 1589. (e) Ueda, J.; Harada, S.; Kobayashi, M.; Yanagawa, M.; Nemoto, T. Eur. J. Org. Chem. 2021, 3999. (11) (a) Kotha, S.; Deb, A. C.; Lahiri, K.; Manivannan, E. Synthesis 2009, 2, 165. (b) Ding, A.; Meazza, M.; Guo, H.; Yang, J. W.; Rios, R. Chem. Soc. Rev., 2018, 47, 5946.

(12) (a) Cai, X.; Keshavarz, A.; Omaque, J. D.; Stokes, B. J. Org. Lett. 2017, 19, 2626. (b) Cai, X.; Tohti, A.; Ramirez, C.; Harb, H.; Fettinger, J. C.; Hratchian, H. P.; Stokes, B. J. Org. Lett. 2019, 21, 1574.

(13) For a discussion of the enhanced nucleophilicity of the para position of fluorobenzenes, see: Rosenthal, J.; Schuster, D. I. J. Chem. Educ. 2003, 80, 679. 